\title{
Multi-parent QTL mapping reveals stable QTL conferring resistance to Gibberella ear rot in maize
}

\author{
Ana L. Galiano-Carneiro (i) Bettina Kessel - Thomas Presterl • \\ David Sewordor Gaikpa (1) Maria Belen Kistner (iD) Thomas Miedaner (i)
}

Received: 17 June 2020/Accepted: 9 December 2020/Published online: 29 December 2020

(C) The Author(s) 2020

\begin{abstract}
Maize production is on risk by Gibberella ear rot (GER) caused by Fusarium graminearum. This is one of the most important ear rot diseases in temperate zones as it leads to yield losses and production of harmful mycotoxins. We investigated, for the first time, the potential use of Brazilian tropical maize to increase resistance levels to GER in temperate European flint germplasm by analyzing six interconnected biparental populations. We assessed GER symptoms in Brazil and in Europe in up to six environments (= location $\times$ year combinations) during the growing seasons of 2018 and 2019. We conducted multi-parent QTL and biparental QTL mapping, and identified four QTLs with additive gene
\end{abstract}

A. L. Galiano-Carneiro - D. S. Gaikpa ·

M. B. Kistner · T. Miedaner $(\square)$

State Plant Breeding Institute, University of Hohenheim, Stuttgart, Germany

e-mail: miedaner@uni-hohenheim.de

A. L. Galiano-Carneiro - B. Kessel · T. Presterl Kleinwanzlebener Saatzucht (KWS) SAAT SE \& Co. KGaA, Einbeck, Germany

M. B. Kistner

Estación Experimental Pergamino, Instituto Nacional de Tecnología Agropecuaria (INTA), CC31,

B2700WAA Pergamino, Argentina

M. B. Kistner

Consejo Nacional de Investigaciones Científicas y

Técnicas (CONICET), Godoy Cruz 2290,

C1425FQB Buenos Aires, Argentina action, each explaining 5.4 to $21.8 \%$ of the total genotypic variance for GER resistance. Among them, QTL q1 was stable across test environments, populations, and between inbred lines and testcrosses. The accuracies of genomic prediction ranged from 0.50 to 0.59 depending on the resistance donor and prediction model. Jointly, our study reveals the potential use of Brazilian resistance sources to increase GER resistance levels by genomics-assisted breeding.

Keywords Gibberella ear rot (GER) - Fusarium graminearum - Stable resistance - Genetic resources · QTL mapping · Genomic selection

\section{Introduction}

Fusarium spp. is one of the most important crop pathogens in maize (Zea mays L.) reducing yield and threatening human and animal health by mycotoxins. These hemibiotrophic fungi can cause diseases during all growth stages of the plant including stalk and ear rots (Munkvold et al. 1997; Pfordt et al. 2020). F. graminearum, $F$. verticillioides, and $F$. temperatum, a new species separated from $F$. subglutinans, are the main species causing ear rot in temperate zones (Pfordt et al. 2020). The composition of species in each environment is mainly associated with weather conditions during silking. At this developmental stage, 
F. graminearum is favored by high precipitation and moderate temperatures whereas $F$. verticillioides is favored by low humidity and high temperatures (Bottalico 1998; Munkvold 2003; Pfordt et al. 2020).

In NW Europe, the main use of maize is for animal feed as silage or corn-cob-mix (Deutsches Maiskomitee 2020). GER symptoms appear as white to pinkish mold starting on the tip of the cobs and may cover the entire cob in a susceptible genotype. F. graminearum produces mainly deoxynivalenol (DON), a mycotoxin causing reduction of voluntary feed intake and even vomiting, and zearalenone (ZEA), an estrogen-like mycotoxin that causes fertility disorders including abortions (Döll and Dänicke 2011). Among livestock, pigs are the main consumers of corn-cob-mix (Deutsches Maiskomitee 2020) and they are the most sensitive animals to these mycotoxins (Pierron et al. 2016). For this reason, the European Union established an orientation value of maximum $0.9 \mathrm{mg}$ DON kg for pig feed (European Commission 2006). However, this limit can be easily surpassed when the environmental conditions are favorable for the sporulation and spread of this pathogen (LSV Bayern 2019). For instance, Europe has a high to severe risk of mycotoxin contamination in animal feed where $83 \%$ of the maize samples were tested positive for DON in recent years (Biomin 2020). Little effort in breeding resistant varieties against ear rot was made in the past and nowadays most of commercial hybrids have a lower ear rot resistance than desirable (Bush et al. 2004; Mesterházy et al. 2012; Zila et al. 2013).

In the European Union, no fungicides are released to control Fusarium diseases in maize and, thus, agronomical practices such as ploughing and host resistance are the most promising methods to control disease spread and mycotoxin accumulation (Bolduan et al. 2009; Pfordt et al. 2020). Reduction of DON accumulation through resistance breeding has been observed for all maize maturity groups (LSV Bayern 2019; Löffler et al. 2009). Furthermore, genotypes with less DON accumulation do not negatively affect the expression of agronomical traits (Martin et al. 2012c), thus allowing breeding of high yielding cultivars (Vigier et al. 2001; Eller et al. 2008; Martin et al. 2012c).

For GER, uniquely quantitative resistance has been identified to date (Martin 2012; Gaikpa and Miedaner 2019). Several studies based on adapted germplasm have been conducted to dissect the genetic architecture of this trait. They identified QTLs explaining 21 to $59 \%$ of the total genotypic variance (Martin et al. 2011, 2012b; Giomi et al. 2016; Han et al. 2016, 2018; Kebede et al. 2016; Gaikpa and Miedaner 2019). Employing exotic germplasm may introduce new sources of resistance alleles to adapted European germplasm (Gaikpa and Miedaner 2019). Tropical and subtropical maize as well as popcorn populations are possible sources of resistant alleles for Fusarium ear rot for temperate maize breeding pools (Zila et al. 2013) and should be explored to achieve higher resistance levels. However, only few studies exploiting genetic resources to increase ear rot resistance have been conducted (Mesterházy et al. 2012; Zila et al. 2013; Butrón et al. 2015).

With the aim to identify QTL with a high environmental stability we evaluated six biparental populations originating from crosses between Brazilian resistant genotypes and European susceptible germplasm. In Brazil, two biparental populations comprising 273 double haploid (DH) lines were tested while four bi-parental populations comprising 486 hybrids were tested in Europe with one common resistance donor being the same. In particular, our objectives were to: (1) validate the use of Brazilian genetic resources in Europe; (2) dissect the genetic architecture of GER resistance in these sources; (3) verify the usefulness of genomics-assisted breeding to boost breeding progress for this complex quantitative trait.

\section{Materials and methods}

\section{Plant material and field trials}

Our experiments comprised six biparental populations: $\mathrm{T} 3 \times \mathrm{A} 6, \mathrm{~T} 3 \times \mathrm{A} 7, \mathrm{~T} 3 \times \mathrm{A} 8, \mathrm{~T} 3 \times \mathrm{A} 12$, $\mathrm{T} 4 \times \mathrm{A} 4$ and T4 $\times$ A5, with 99, 174, 155, 71, 110, and 150 individuals, respectively, each resulting from a cross between a GER resistance donor (Brazilian tropical DH line, "T") and a GER susceptible recipient (European adapted DH line, "A”). Recipients "A6", "A7", "A8" and "A12" belong to the stiff stalk synthetic (SSS) while recipients "A4" and "A5" belong to the non-stiff stalk (NSSS) heterotic groups, respectively. Populations $\mathrm{T} 3 \times \mathrm{A} 6$ and $\mathrm{T} 3 \times \mathrm{A} 7$ comprised 99 and 174 double haploid (DH) lines, respectively, and were assessed in Brazil as line per se in Campo Largo in 2018 and in Ponta Grossa in 2019, 
both cities in Paraná state located in the southern region of Brazil. Jointly, 486 testcrosses from the other four biparental populations were evaluated in Europe in three locations: Monselice, Italy, and Gondelsheim and Bernburg, Germany, during the growing seasons of 2018 and 2019 (except by donor T4 which was assessed uniquely in 2019) leading to up to six testing environments, $(=$ combination of location $\times$ year $)$. All progenies intended to be tested in Europe were crossed with the same susceptible early flint tester aiming to establish chilling tolerance and an earlier maturity for the European testing locations. For simplification, we will refer to $\mathrm{T} 3 \times \mathrm{A} 6$ and T3 $\times$ A7 as T3 donor, tested in Brazil as per se populations, to $\mathrm{T} 3 \times \mathrm{A} 8$ and $\mathrm{T} 3 \times \mathrm{A} 12$ as $\mathrm{T} 3$ donor, and T4 $\times$ A4 4 and T4 $\times$ A5 as T4 donor, both tested in Europe as testcross populations.

Our experiments were allocated in an alpha design with two replications where each experimental unit comprised a four-meter row with approximately 20 plants. Standard agricultural practices including insecticides and fungicides not being effective against Fusarium were applied at the Brazilian locations.

Inoculation and trait assessment

In Brazil, inoculum was kindly provided by Dr. Lygia Vitória Galli Terasawa (Federal University of Paraná, Curitiba, Brazil). The inoculum was obtained by isolating three sources of Fusarium graminearum from contaminated maize cobs collected at three different locations in the state of Paraná, Brazil, in the growing seasons 2015 and 2016. An inoculum suspension with a concentration of 50,000 conidia ml ${ }^{-1}$ containing these three inoculum sources was produced and $1 \mathrm{ml}$ was inoculated into the maize silk channel. In Europe, the highly aggressive Fusarium graminearum strain IFA 66 was kindly provided by Prof. Dr. Marc Lemmens (University of Natural Resources and Life Sciences, Vienna, Austria) and used to prepare our inoculum suspension following the protocol of Reid et al. (1996). Two $\mathrm{ml}$ of the inoculum suspension containing $1.5 \times 10^{4}$ spores $\mathrm{ml}^{-1}$ were applied with a one-needle vaccinator on the silk channel of the maize cobs in the European locations. Both in Brazil and in Europe, ten plants of each experimental unit were inoculated, excluding the first plant of the row due to possible border effect, three to 6 days after the experimental unit was flowering. Rows were declared as flowering when at least $50 \%$ of the plants on the row presented extruded silks. Female flowering (FF) dates were collected for each row in a two-day interval.

Approximately 50 days after inoculation, cobs were dehusked and all plants were visually assessed for GER symptoms by estimating the percentage of the ear covered by mycelium (Fig. 1). The 10 noninoculated plants were used as a control of the proportions of naturally infected cobs. The arithmetic means of the 10 assessed inoculated and the 10 control plants (= naturally infected), respectively, were employed for further statistical analyses.

\section{Phenotypic data analysis}

Phenotypic analyses for single environments were performed using linear mixed models and outlier detection procedures as proposed by Bernal-Vasquez et al. (2016). All GER phenotypic data were arcsine square root transformed to attend the normality assumption and reduce the heterogeneity of variances. Combined analysis without critical outliers (not more than $15 \%$ of the complete data were removed) were conducted according to the following mixed model:

$$
\begin{aligned}
y_{i j k l m}= & \mu+G_{i}+Y_{j}+L_{k}+L Y_{k j}+L Y R_{k j l}+L Y R B_{k j l m} \\
& +e_{i j k l m}
\end{aligned}
$$

where $y_{i j k l m}$ was the phenotypic observation of the $i$ th genotype, $j$ th year, $k$ th location, $l$ th replication and $m$ th incomplete block. The symbol $\mu$ represents the overall mean, $G_{i}$ the effect of the $i$ th genotype, $Y_{j}$ is the effect of the $j$ th year, $L_{k}$ the effect of the $k$ th location, and its interaction terms, $R_{l}$ is the effect of the $l$ th replication, $B_{m}$ the effect of the $m$ th incomplete block, and $e_{i j k l m}$ is

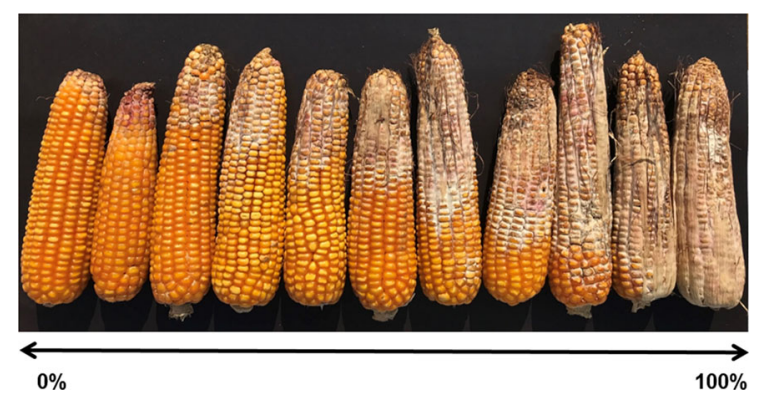

Fig. 1 Assessment scale of damaged maize cobs by GER. 0\% represents healthy and $100 \%$ completely diseased cobs. The percentage is assigned depending on the percentage of the cob with GER symptoms 
the heterogeneous error variance. The same model excluding the year effects was used for the single location analysis.

$G_{i}$ and $Y_{j}$ effects were included in the fixed statement of the model to obtain the best linear unbiased estimators (BLUEs). The variance components were obtained through the restricted maximum likelihood method (REML) by including only the $Y_{j}$ effects in the fixed statement of the model above. The significance of the variance components was obtained by likelihood ratio test between the full and incomplete model (Stram and Lee 1994). Binary dummy variables were used to separate the effects of each population, checks and replicates as proposed by Piepho et al. (2006). For the sake of simplicity, they were not shown in the described model.

The broad sense heritability $\left(\mathrm{H}^{2}\right)$ was estimated following the formula:

$H^{2}=\frac{\sigma_{G}^{2}}{\sigma_{G}^{2}+\frac{\sigma_{L}^{2}}{L}+\frac{\sigma_{Y}^{2}}{Y}+\frac{\sigma_{L Y}^{2}}{L Y}+\frac{\sigma_{e}^{2}}{L Y R}}$

where $\sigma_{G}^{2}, \sigma_{L}^{2}, \sigma_{Y}^{2}, \sigma_{L Y}^{2}$ and $\sigma_{e}^{2}$ are the genotypic, location, year, location $\times$ year and error variances, respectively. $L, Y$ and $R$ correspond to the number of locations, years, and replicates, respectively. Phenotypic correlations based on BLUEs were calculated with Pearson product moment correlation coefficients.

In the inbred populations tested in Brazil, the correlation coefficient between GER and FF was significant $(\mathrm{r}=-0.49$ and $p<0.001$ ) (data not shown). Therefore, GER was adjusted for FF by including $\mathrm{FF}$ as fixed effect in the mixed model to estimate the best linear unbiased estimators (BLUEs) as described by Emrich et al. (2008). After the corrections, the correlation between GER and FF was reduced to $\mathrm{r}=-0.30(p<0.001)$. This GER rating adjusted for FF (GER_FF) was used for all further analysis. In the testcross populations tested in Europa, the correlation coefficient between GER and FF was low and not significant $(r=-0.033$ and $p>0.05$ ), therefore no corrections for FF were necessary.

All analyses were conducted in $\mathrm{R}$ environment $(\mathrm{R}$ Development Core Team 2018, version 3.5.1). Mixedmodel computations were performed by using ASReml-R 3.0 (Gilmour et al. 2009).
Molecular data

DH lines were genotyped at KWS molecular laboratory with an Illumina $15 \mathrm{k}$ SNP chip based on the public Illumina MaizeSNP50 BeadChip. The ten maize chromosomes were partitioned into bins of $0.5 \mathrm{cM}$ (genetic map IBM, physical map AGPv02, Ganal et al. 2011) to construct the genetic map. Regions adjacent to centromers were especially markedly enriched to account for the low recombination rates in this chromosome area.

The number of polymorphic markers in each population ranged from 5832 to 7039 . Quality control was conducted by removing monomorphic or missing alleles for both parents, genotypes with more than $25 \%$ missing values, markers with more than $10 \%$ missing data and markers with minor allele frequency (MAF) lower than $5 \%$ in each population. After the quality check, 4603, 5585 and 2784 SNP markers were available for the Brazilian crosses with $\mathrm{T} 3$, the European crosses with T3 and T4, respectively.

QTL mapping analysis

Multi-parent QTL mapping analysis was conducted with the R package mppR version 1.2.1 (Garin et al. 2018). By employing this package, interconnected biparental populations from each continent were analyzed jointly by the method of composite interval mapping (CIM) (Zeng 1993, 1994). We obtained the allele-substitution effect of the identified QTL through a bi-allelic model where alleles from different populations are considered to be identical by state (IBS), same SNP score transmitted the same allele for all individuals with common parents (e.g., model B in Würschum et al. 2012; Garin et al. 2017). For this model, population structure was accounted by the k-model proposed by $\mathrm{Yu}$ et al. (2006).

Permutation tests were conducted by performing 1000 iterations and the significance thresholds were obtained from the 90th percentile of the maximum LOD score distribution of all iterations (Broman and Sen 2009). QTL mapping for each model was conducted in a first step by a simple interval mapping (SIM) and the significant QTL from this analysis were applied as cofactors for the CIM. The confidence interval of each QTL was obtained by $-\log 10(p)$ value drop off interval. The contribution of each QTL to the phenotypic variance was computed by 
comparing the full model, containing all the QTL, and the incomplete model, excluding only the detected QTL of interest. Individual explained genotypic variance $\left(p_{G}\right)$ were obtained following the equation proposed by Utz et al. (2000):

$p_{G}=\frac{R_{a d j}^{2}}{H^{2}}$

where $R_{a d j}^{2}$ corresponds to the adjusted $R^{2}$ from the linear model containing all identified QTL and $H^{2}$ to the average heritability of heritability estimates for individual populations with a common donor.

Biparental QTL mapping for population T3 $\times$ A8 was also evaluated individually with the software for meta-QTL analysis (PlabMQTL) (Utz 2011) by the CIM method, as population $\mathrm{T} 3 \times \mathrm{A} 12$ was not included in the QTL analysis due to the low genetic variance. Additive and additive by additive epistatic models were investigated. Empirical thresholds for LOD scores were determined using 1000 permutation tests and assuming an experiment-wise error of 0.10 . The selection of cofactors was done according to the modified BIC (mBIC) model (Baierl et al. 2006). The identified QTL were assumed as co-located when their confidence intervals overlapped.

Marker-assisted, genomic and weighted genomic predictions

Marker-assisted predictions were conducted for breeding values of testcrosses with all QTLs. Genomic prediction was carried out by ridge-regression BLUP (RR-BLUP; Whittaker et al. 2000) with the R package "rrBLUP', (Endelman 2011; Endelman and Jannink 2012) within each donor group. Missing SNP marker information was imputed for each donor group with the software LinkImpute (Money et al. 2015) and resulted in high imputation accuracies $(>90 \%)$. In addition, we performed a weighted ridge-regression BLUP (wRR-BLUP) where the same significant markers applied for marker-assisted predictions were included in the fixed statement of the genomic prediction model (Zhao et al. 2014; Spindel et al. 2016). The prediction accuracy was defined as the Pearson's product-moment correlation coefficient between observed and predicted trait values divided by the square root of the broad-sense heritability.

\section{Results}

Adjusted means for GER severity ranged, on average, from 4.9 to 10.0 for per se populations and 24.4 to $28.9 \%$ for the testcross populations (Table 1). Entrymean heritabilities were moderate to high ranging from 0.68 to 0.72 for per se populations and 0.44 to 0.72 for testcross populations except for population T3 $\times$ A12 where the heritability was only 0.24 due to the non-significant genetic variation (Table 1). For this reason, the population $\mathrm{T} 3 \times \mathrm{A} 12$ was not included in the QTL mapping analysis. Both GER_FF and GER showed a quantitative distribution with T3 being more resistant than the adapted parental lines (Fig. 2). Within Europe, most of the locations showed higher GER severity in 2019 compared to 2018 and this tendency was observed for all biparental populations (Fig. 3).

Jointly, we identified four QTL that explained 5.4 to $21.8 \%$ of the genetic variance, most of them had minor effects $\left(<15 \% p_{G}\right)$ only. They were located on chromosome bins 1.02, 3.08, 5.06, and 8.05. No dominance or additive $\times$ additive QTL were identified indicating uniquely additive QTL for GER_FF and GER in our study. QTL q1 was identified across all QTL analyses performed including different biparental populations, and line and testcross populations across both continents. QTL q1 explained between 10.2 and $21.8 \%$ of the genotypic variance where the highest variance was observed for population T3 $\times$ A8 (Table 2). Moreover, none of the identified QTL for GER were overlapping with the identified QTL for FF (data not shown).

Prediction accuracy by weighted genomic prediction (wRR-BLUP) was slightly higher compared to marker-assisted selection for both donors (MAS, Fig. 4). Prediction accuracy for GER in testcrosses with donor T3 were of $0.53,0.50$ and 0.59 estimated for MAS, RR-BLUP and wRR-BLUP, respectively. Lower prediction accuracy for MAS (0.47), wRRBLUP (0. 57) and RR-BLUP (0.55) was obtained for testcrosses with donor T4. For both donors, the wRRBLUP method led to the highest prediction accuracies. For population $\mathrm{T} 3 \times \mathrm{A} 8$ only $w$ RR-BLUP led to slightly improved predictions compared to MAS (data not shown). 
Table 1 Statistics summary and variance components for GER_FF (arcsin transformed Gibberella ear rot adjusted for female flowering date, original data without transformation in parentheses) of two populations of $\mathrm{DH}$ inbreds evaluated in Brazil and GER (arcsin transformed Gibberella ear rot) of testcrosses of four DH inbred populations evaluated in Europe

\begin{tabular}{|c|c|c|c|c|c|c|}
\hline \multirow{2}{*}{$\begin{array}{l}\text { Trait } \\
\text { Pop } \\
\text { No. env. }\end{array}$} & \multicolumn{3}{|l|}{ Brazil } & \multicolumn{3}{|l|}{ Europe } \\
\hline & $\begin{array}{l}\text { T3xA6 } \\
2\end{array}$ & T3xA7 & $\begin{array}{l}\text { T3xA8 } \\
6\end{array}$ & T3xA12 & $\begin{array}{l}\text { T4xA4 } \\
3\end{array}$ & T4xA5 \\
\hline \multicolumn{7}{|c|}{ Phenotypic data } \\
\hline Mean & $4.94(7.66)$ & $10.01(10.99)$ & $27.81(31.20)$ & $28.86(33.45)$ & $28.61(31.99)$ & $24.33(27.63)$ \\
\hline Median & $3.25(2.64)$ & $8.00(8.30)$ & $27.96(31.50)$ & $27.80(31.52)$ & $28.23(30.57)$ & $23.65(26.60)$ \\
\hline Min & $0.16(0.00)$ & $0.00(0.00)$ & $5.23(6.83)$ & $10.70(11.49)$ & $4.12(5.05)$ & $5.89(6.25)$ \\
\hline Max & $47.56(51.13)$ & $76.24(77.50)$ & $59.26(55.00)$ & $79.30(77.99)$ & $62.41(63.49)$ & $62.96(61.75)$ \\
\hline $\mathrm{LSD}_{5} \%$ & 2.38 & 2.38 & 4.34 & 4.34 & 4.34 & 4.34 \\
\hline $\mathrm{n}$ & 99 & 174 & 155 & 71 & 110 & 150 \\
\hline \multicolumn{7}{|c|}{ Variance components } \\
\hline$\sigma_{G}^{2}$ & $0.01 * * *$ & $0.04 * * *$ & $0.010 * * *$ & 0.002 & $0.007 * * *$ & $0.007 * * *$ \\
\hline$\sigma_{G \times L}^{2}$ & $0.01 * * *$ & $0.01 * * *$ & 0.001 & 0 & $0.012 * * *$ & $0.003 *$ \\
\hline$\sigma_{G \times Y}^{2}$ & - & - & 0 & 0 & - & - \\
\hline$\sigma_{G \times Y \times L}^{2}$ & - & - & $0.008^{* *}$ & $0.018 * * *$ & - & - \\
\hline$\sigma_{e}^{2}$ & 0.01 & 0.01 & 0.060 & 0.060 & 0.03 & 0.03 \\
\hline $\mathrm{H}^{2}$ & 0.72 & 0.68 & 0.61 & 0.24 & 0.44 & 0.54 \\
\hline
\end{tabular}

Gibberella ear rot was estimated as the percentage of ear affected. Minimum (Min), median, mean, and maximum (Max.) scores are shown for the backtransformed phenotypic data. Number of genotypes (n) and least square of a difference (LSD5\%) are also indicated. The variance components include the genetic $\left(\sigma_{\mathrm{G}}^{2}\right)$, genotype-location $\left(\sigma_{\mathrm{G} \times \mathrm{L}}^{2}\right)$, genotype-year $\left(\sigma_{\mathrm{G} \times \mathrm{Y}}^{2}\right)$, genotype-yearlocation interactions $\left(\sigma_{\mathrm{G} \times \mathrm{Y} \times \mathrm{L}}^{2}\right)$, and residuals $\left(\sigma_{\mathrm{e}}^{2}\right)$ variances. Entry mean heritability $\left(\mathrm{H}^{2}\right)$ for each population are also assigned $* p<0.05 ; * * p<0.01 ; * * * p<0.001$
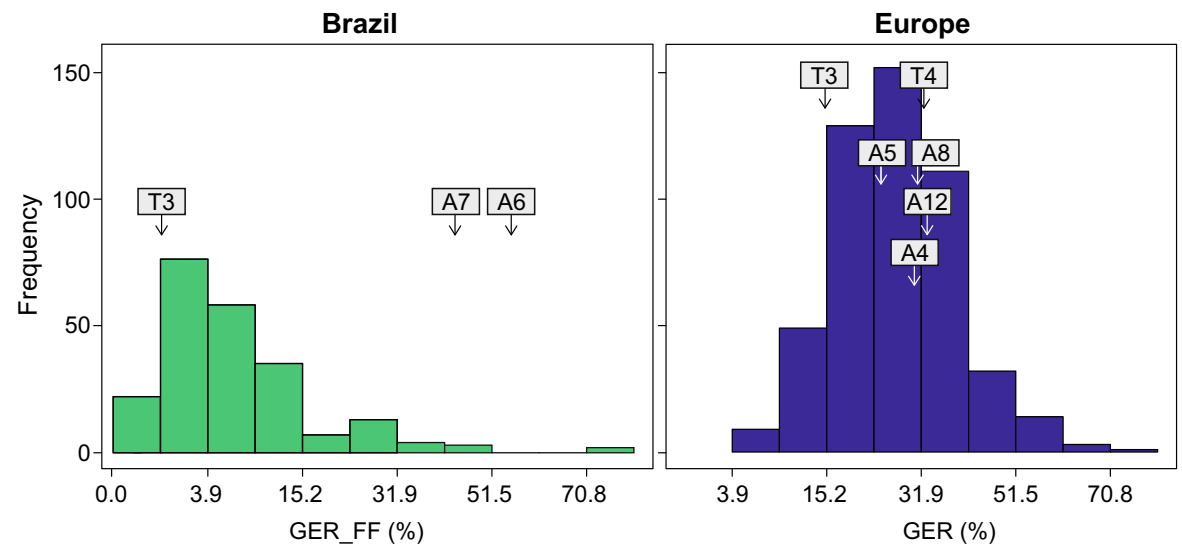

Fig. 2 Phenotypic distribution of the backtransformed Gibberella ear rot (GER) data assessed in Brazil adjusted for female flowering date (FF) and in Europe; pointing the respective tropical (T) and adapted (A) parental lines by arrows

\section{Discussion}

The extension of maize acreage to attend the increasing demand in combination with short crop rotations including the Fusarium susceptible wheat will increase the risk of ear rots by Fusarium spp. and subsequent mycotoxin contamination in the near future (Ray et al. 2013; Pfordt et al. 2020). To keep 
Fig. 3 Box plots for Gibberella ear rot (GER) severity (backtransformed values) of different biparental populations evaluated in Europe in six environments (year-location combinations, environments:

GON = Gondelsheim/DE, $\mathrm{BBG}=$ Bernburg/DE, MCE = Monselice/IT; in 2018 and 2019). Horizontal lines within boxes indicate the median, black squares refer to outliers. The checks comprised parental lines and commercial resistant and susceptible hybrids

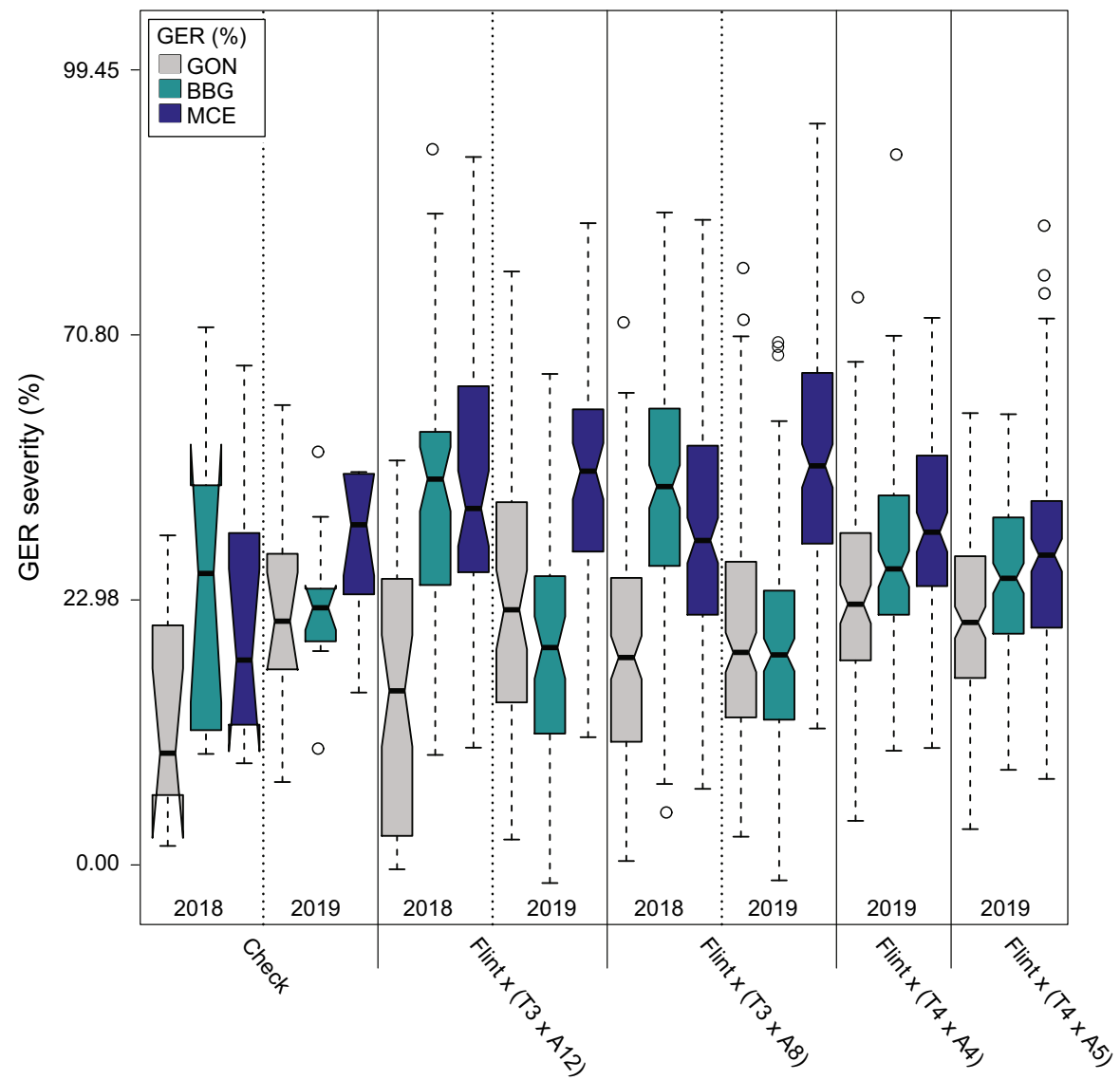

Table 2 QTL for Gibberella ear rot resistance identified across populations sharing the same inbred donor and inbreeding level (multi-parent QTL mapping

\begin{tabular}{|c|c|c|c|c|c|c|c|c|c|c|}
\hline Population & $\mathrm{n}_{\mathrm{G}}$ & $\mathrm{n}_{\mathrm{M}}$ & Type & QTL & Bin & QTL (cM) & Range (cM) & LOD score & $p_{G}(\%)$ & $\alpha$-effect \\
\hline \multicolumn{11}{|l|}{ Brazil } \\
\hline T3 × A6_A7 & 266 & 4603 & PS & q1 & 1.02 & 60.54 & $58.89-62.92$ & 5.57 & 10.17 & -0.96 \\
\hline T3 × A6_A7 & 266 & 4603 & PS & $\mathrm{q} 2$ & 3.08 & 196.72 & 194.99-197.03 & 4.63 & 14.86 & -1.33 \\
\hline T3 × A6_A7 & 266 & 4603 & PS & q3 & 5.06 & 162.53 & $161.56-162.71$ & 4.65 & 5.37 & -0.43 \\
\hline \multicolumn{11}{|l|}{ Europe } \\
\hline T4 × A4_A5 & 229 & 2784 & $\mathrm{TC}$ & $\mathrm{q} 1$ & 1.02 & 58.64 & $50.40-85.62$ & 3.74 & 10.92 & 0.35 \\
\hline T4 × A4_A5 & 229 & 2784 & $\mathrm{TC}$ & $\mathrm{q} 4$ & 8.05 & 120.04 & $119.75-120.56$ & 3.78 & 11.67 & 0.35 \\
\hline $\mathrm{T} 3 \times \mathrm{A} 8$ & 145 & 5585 & $\mathrm{TC}$ & $\mathrm{q} 1$ & 1.02 & 60.00 & 59.93-61.04 & 6.56 & 21.84 & -0.34 \\
\hline
\end{tabular}

Populations T3 $\times$ A6 and T3 $\times$ A7 were written as T3 $\times$ A6_A7 for simplification, as well as populations T4 $\times$ A4 and T4 $\times$ A5, T4 $\times$ A4_A5) or T3 $\times$ A8 (with PLABMQTL), number of genotypes $\left(\mathrm{n}_{\mathrm{G}}\right.$ ), number of markers used ( $\mathrm{n}_{\mathrm{M}}$ ), type of population assessed (PS for per se and TC for testcrosses), QTL location (cM), QTL confidence interval range (cM), explained genotypic variance $(p G)$ and the backtransformed allele substitution effect ( $\alpha$-effect) of the tropical parent for GER_FF assessed in Brazil and GER assessed in Europe. Bolded name indicates co-located QTL

resistance levels high in the long-term, it is essential to employ diversified resistance sources (Nelson et al.
2018). Tropical maize, including Brazilian germplasm, could be valuable sources of resistance alleles 


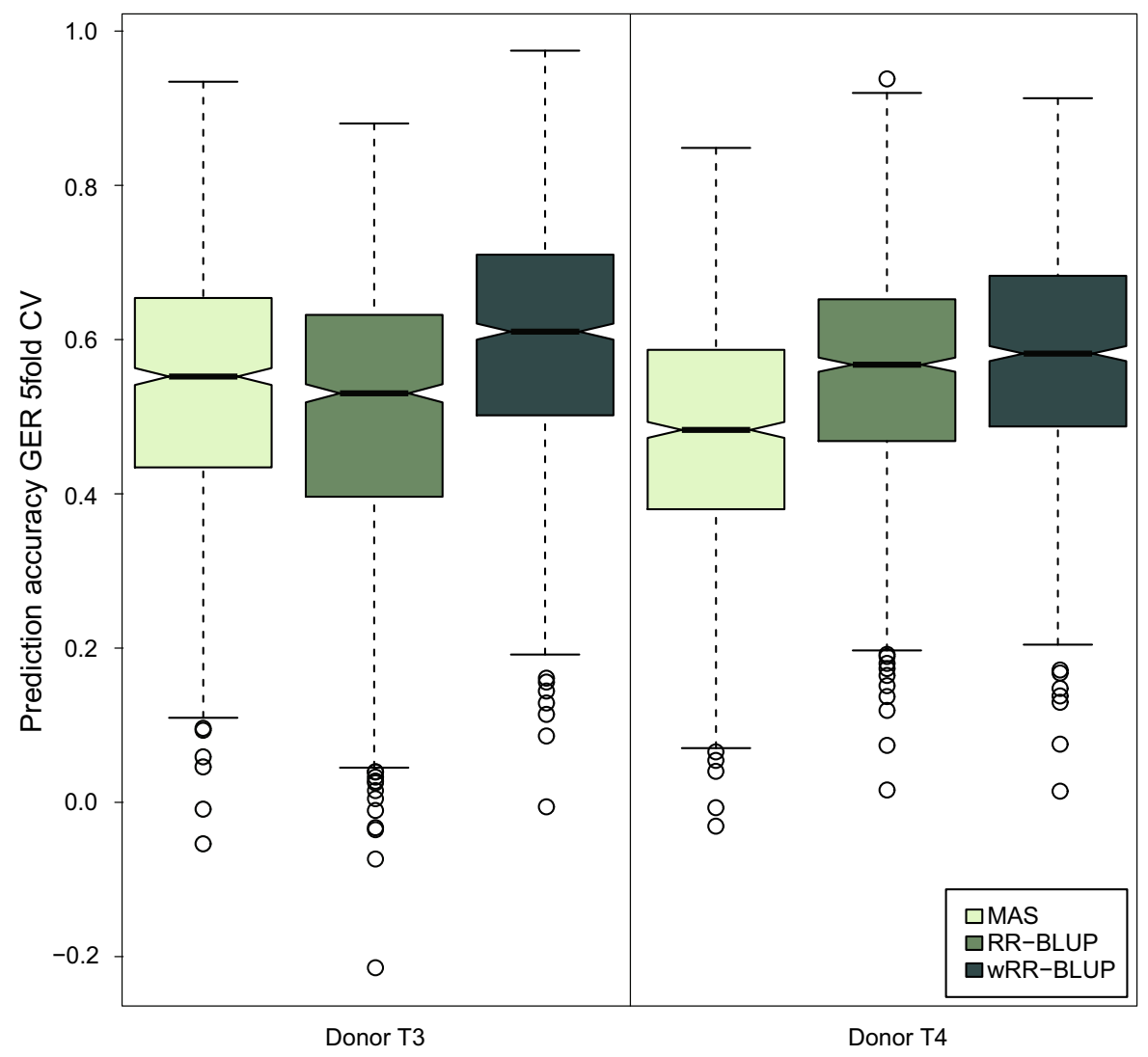

Fig. 4 Prediction accuracies obtained from marker assisted selection (MAS), genomic selection (RR-BLUP) and weighted genomic selection (wRR-BLUP) for each donor group and continent for testcrosses

for temperate germplasm (Hallauer et al. 2010; Poland et al. 2011), but are not yet fully exploited. Therefore, we investigated the potential use of Brazilian sources as GER resistance donors for European flint maize. Aiming for environmentally stable resistances we tested a total of six interconnected biparental populations both in Brazil and in Europe.

\section{Assessing GER in contrasting environments}

In Brazil, tropical parent "T3" showed higher resistance levels compared to the European adapted parents "A6" and "A7" as expected. However, the population mean for GER damage was low in both environments. This could be explained by the concentration of spores. We applied 50,000 spores $\mathrm{ml}^{-1}$ in each maize ear in the experiments located in Brazil, but an even higher concentration might be necessary to increase disease severity. Still, genetic variance was significant with moderate to high heritabilities. Conversely, in
Europe, the genetic variance was lower than in Brazil and only the tropical parent T3 was more resistant than the adapted lines. The tropical parent T4 and adapted European parent inbred lines, however, were similarly susceptible.

Our phenotypic data was assessed after inoculation of maize cobs through the silk channel. This is the most important infection pathway for $F$. graminearum in the absence of insect injury and the most common in the northern maize growing regions (Reid et al. 1992, 1996; Munkvold et al. 1997; Bolduan et al. 2009). However, this method has the disadvantage to be unstable across different weather conditions (Reid et al. 1996; Mesterházy et al. 2012; Butrón et al. 2015). This can be one of the reasons why the GER severity was lower in 2018 compared to 2019 for most of the European locations.

The genotype $\times$ environment interactions were high and significant both in Brazil and in Europe. This is in accordance with other studies where 
resistance was found to be variable when assessing GER resistance in several contrasting environments (Bolduan et al. 2009; Löffler et al. 2009). Independent selection for each geographic region was recommended (Butrón et al. 2015) and is practiced in Europe according to the different breeding programs assigned to each maturity group. In our study, we assessed phenotypic data in up to six contrasting environments as the main objective of this research work was to identify stable resistance QTL that are effective even in the current global warming conditions.

QTL mapping reveals stable QTL

across continents, environments, and populations

We identified four QTL explaining between 5.37 and $21.84 \%$ of the GER genotypic variance where most of them had minor effects $\left(<15 \% p_{G}\right)$. This is in accordance with other studies that identified many QTL with small effects and a global explained genotypic variance varying between $21 \%$ and $59 \%$ for GER resistance (Martin et al. 2011, 2012b; Kebede et al. 2016; Gaikpa and Miedaner 2019). Martin et al. (2012a) identified QTL explaining between 21 and $49 \%$ of the global genotypic variance in three biparental European populations with no common QTL identified across populations. QTL q1 was identified across populations. In addition, this QTL had a major effect on population T3 $\times$ A 8 and could alone explain $21.8 \%$ of the genotypic variance. The favorable allele originated from the tropical parent $\mathrm{T} 3$ indicating that this Brazilian donor can be a great source of stable QTL for GER resistance. However, we identified only a low number of QTL. This might indicate that possibly each family was segregating for a different set of QTLs and/or other genomic regions conferring resistance to GER could not be identified due to the highly quantitative nature of this trait (Blanc et al. 2006; Ogut et al. 2015; Han et al. 2016). However, we also conducted a QTL mapping for each family separately (data not shown) and did not identify a larger number of QTL. Another main reason might be that only few QTL are stable across six European environments including two very contrasting years and field locations (northern Italy and Germany). This conclusion is supported by the high genotype $\times$ environment interaction variances.

QTL conferring GER resistance were identified on chromosome bins 1.02, 3.08, 5.06 and 8.05 in our study, namely QTL q1, q2, q3 and q4, respectively (Table 1). The QTL q1 identified across environments and populations is located in a genomic region known to confer resistance to ear rot caused by multiple pathogens (Wisser et al. 2006). QTL q2 was identified in the same bin position previously reported to significantly contribute to GER resistance and reduced DON contamination, while the QTL on chromosome bin 5.06 was in the same bin as a QTL previously reported to be associated to DON contamination (Martin et al. 2012; Martin et al. 2012b). Kebede et al. (2016) identified one QTL for GER resistance near the QTL identified on chromosome bin 8.05. Overlapping QTL between GER and DON are expected as both traits are highly correlated ( $r$ > 0.86; Butrón et al. 2015; Miedaner et al. 2015). This was confirmed by co-located QTL for GER resistance and reduced DON contamination in QTL mapping studies suggesting that both traits are likely to be controlled by a set of the same genes (Martin et al. 2012b; Han et al. 2016). Additionally, different genes might also play a role in GER resistance and reduced DON accumulation (Gaikpa and Miedaner 2019).

Our germplasm included families belonging to the SSS and NSSS heterotic groups, comprising populations of donors T3 and T4, respectively. We identified a larger number of QTL within the SSS group compared to the NSSS, but this is probably due to the unbalanced number of families per heterotic group in our study with four families from SSS and two from NSSS, and the GER severity discrepancy between the parental components of each heterotic group. Conversely, other studies identified that the flint germplasm was more susceptible to ear rot and showed higher DON and ZEA concentrations compared to the dent pool. These differences were assigned to the few founding populations composing the flint pool compared to the dent pool which had a constant influx from germplasms from other regions (Reif et al. 2005; Löffler et al. 2010).

The major infection pathway of $F$. graminearum is via the silks, but some species such as $F$. verticillioides can infect cobs after silking additionally via insect injuries on the cobs (Reid et al. 1992, 1996; Pfordt et al. 2020). Kebede et al. (2016) investigated infection by $F$. graminearum both through silk and kernels and identified only three QTLs overlapping for both infection pathways. These co-located QTL were 
identified on chromosomes 1, 2, and 8, where the QTL on chromosome 8 was identified in a close location to our QTL q4 (Kebede et al. 2016). With rising temperatures due to global climatic change damage by insects might increase in frequency and severity, especially in the tropics and subtropics (Juroszek and von Tiedemann 2013). For this reason, the identification of QTL that are common among different infection pathways can lead to a broader resistance.

In summary, the QTL identified in our study showed mainly additive effects and no additive $\times$ additive epistasis. This is in accordance with other studies where GER was found to be controlled by several additive QTL (Martin et al. 2012a) and epistatic gene effects were of little importance in most of the testing environments (Butrón et al. 2015). Therefore, mainly additive and dominance effects should be considered in a breeding program aiming to increase ear rot resistances and decrease mycotoxin accumulation (Butrón et al. 2015). In a study of GER resistance in maize, mid-parent heterosis was observed indicating partial dominance (Martin et al. 2012c). This is in accordance to results of Gendloff et al. (1986) and Chungu et al. (1996) who identified dominance and dominance $\times$ dominance gene action although additive effects were more important.

Genomics-assisted breeding can successfully select superior resistant genotypes for GER

QTL q1 alone explained 21.8\% of genetic variation for GER in testcrosses of the mapping population $\mathrm{T} 3 \times \mathrm{A} 8,10.2 \%$ across per se populations derived from $\mathrm{T} 3 \times \mathrm{A} 6$ and $\mathrm{T} 3 \times \mathrm{A} 7$, and $10.9 \%$ across testcrosses of the mapping populations derived from T4 $\times$ A4 and T4 $\times$ A5. Therefore, genomic selection did not lead to a significantly higher prediction accuracy compared to the marker assisted selection approach (Fig. 4). It is important to notice that our prediction accuracies might be overestimated as the same germplasm was composing both the training and prediction sets. In addition, the phenotypic data of all genotypes were collected in the same environments which may not illustrate the reality of commercial breeding programs. Moreover, before the application of the identified QTLs in MAS a QTL validation is necessary. Brauner et al. (2016) conducted the first validation study for QTLs on GER resistance. They tested six QTL identified in a previous mapping study and introgressed them into two different genetic backgrounds. Resistance alleles at three QTLs significantly increased resistance to GER, but the effects were significant only for a small subset of lines due to linkage drag and/or epistasis with residual loci in nontarget regions.

To date, only two studies conducted a genomic selection for GER resistance in maize (Gaikpa and Miedaner 2019). Riedelsheimer et al. (2013) investigated the influence of the training set (TS) composition on the prediction accuracy of agronomic traits and GER on five interconnected biparental DH populations. They identified a decline on prediction accuracy when full-sibs were replaced by half-sibs in the TS. In our analysis, the prediction accuracy of genomic selection was slightly higher for donor T3, for which the TS was composed by the same families of the validation set. The TS of donor T4 was composed by two biparental populations with one common tropical line and had slightly lower predictions than donor T3 ( 0.50 for T4 and 0.55 for T3). Han et al. (2018) reported that increasing the TS set size with genetically distant individuals, in this case of the opposite heterotic group, did not improve the genomic prediction of GER resistance.

\section{Conclusions}

In this research project we tested two Brazilian lines as resistance donors of GER. The tropical parent T3 was resistant even in northern Italian and German locations illustrating the independence of this resistance source from environment. QTL q1 was proven to be stable across populations and continents explaining 10.2 to $21.8 \%$ of the genotypic variance of GER resistance depending on the situation. An independent validation of this QTL would be very valuable. In addition, genomics-assisted breeding can boost selection for GER resistance by wRR-BLUP. Given the different maturity groups and other adaptation problems of tropical germplasm, however, marker-assisted backcrossing of $\mathrm{q} 1$ might be recommendable to integrate this prominent QTL into adapted European germplasm.

Acknowledgements Open Access funding enabled and organized by Projekt DEAL. We greatly acknowledge Dr. Hans Peter Maurer and Dr. Tobias Würschum for their 
phenotypic and molecular analyses advices. We also highly appreciate the excellent technical support of Dr. Francisco Terasawa Jr., Vania Kulka Portes and Alex Viana Alves in Brazil, and Angela Belluco, Eva Marsollek, Dorothee Seyfang, Giulia Andolfo, Hada Cehic, Maria Bakendorf, Marzia Brigato, and Rossella Zerbetto in Europe.

Authors' contributions TM, TP and BK planned the experiments and supervised the project. BK, DSG and MBK supported data collection. AG collected phenotypic data, conducted all statistical analyses, and wrote the manuscript, TM edited it. All authors read the final version for publication.

Funding This project was funded by the Federal Ministry of Food and Agriculture (BMEL) based on a decision of the Parliament of the Federal Republic of Germany via the Federal Office for Agriculture and Food (BLE) under the innovation support program within the PRIMA cooperative project (Grant No. 2818202815). KWS SAAT SE \& Co. KGaA, Einbeck, Germany, provided all genetics materials and conducted all field experiments in the European and Brazilian locations.

\section{Compliance with ethical standards}

Conflict of interest The authors declare that they have no conflict of interest.

Open Access This article is licensed under a Creative Commons Attribution 4.0 International License, which permits use, sharing, adaptation, distribution and reproduction in any medium or format, as long as you give appropriate credit to the original author(s) and the source, provide a link to the Creative Commons licence, and indicate if changes were made. The images or other third party material in this article are included in the article's Creative Commons licence, unless indicated otherwise in a credit line to the material. If material is not included in the article's Creative Commons licence and your intended use is not permitted by statutory regulation or exceeds the permitted use, you will need to obtain permission directly from the copyright holder. To view a copy of this licence, visit http://creativecommons.org/licenses/by/4.0/.

\section{References}

Baierl A, Bogdan M, Frommlet F, Futschik A (2006) On locating multiple interacting quantitative trait loci in intercross designs. Genetics 173:1693-1703. https://doi. org/10.1534/genetics.105.048108

Bayern LSV (2019) DON-Ergebnisse Körnermais. In: LfL, Freising. https://www.lfl.bayern.de/ipz/mais/025515/ index.php. Accessed 8 Jan 2020

Bernal-Vasquez AM, Utz HF, Piepho HP (2016) Outlier detection methods for generalized lattices: a case study on the transition from ANOVA to REML. Theor Appl Genet 129:787-804. https://doi.org/10.1007/s00122-016-2666-6

Biomin (2020) World mycotoxin survey: impact 2020. https:// www.biomin.net/science-hub/world-mycotoxin-surveyimpact-2020/. Accessed 23 Apr 2020
Blanc G, Charcosset A, Mangin B et al (2006) Connected populations for detecting quantitative trait loci and testing for epistasis: an application in maize. Theor Appl Genet 113:206-224. https://doi.org/10.1007/s00122-006-0287-1

Bolduan C, Miedaner T, Schipprack W et al (2009) Genetic variation for resistance to ear rots and mycotoxins contamination in early European maize inbred lines. Crop Sci 49:2019-2028. https://doi.org/10.2135/cropsci2008.12. 0701

Bottalico A (1998) Fusarium diseases of cereals: species complex and related mycotoxin profiles, in Europe. J Plant Pathol 80:85-103

Brauner PC, Melchinger AE, Schrag TA et al (2016) Low validation rate of quantitative trait loci for Gibberella ear rot resistance in European maize. Theor Appl Genet 130:1-12. https://doi.org/10.1007/s00122-016-2802-3

Broman KW, Sen Ś (2009) A guide to QTL mapping with R/qtl. Springer, Madison

Bush BJ, Carson ML, Cubeta MA et al (2004) Infection and fumonisin production by Fusarium verticillioides in developing maize kernels. Phytopathology 94:88-93. https://doi.org/10.1094/PHYTO.2004.94.1.88

Butrón A, Reid LM, Santiago R et al (2015) Inheritance of maize resistance to Gibberella and Fusarium ear rots and kernel contamination with deoxynivalenol and fumonisins. Plant Pathol 64:1053-1060. https://doi.org/10.1111/ppa. 12351

Chungu C, Mather DE, Reid LM, Hamilton RI (1996) Inheritance of kernel resistance to Fusarium graminearum in maize. J Hered 87:382-385. https://doi.org/10.1093/ oxfordjournals.jhered.a023019

Deutsches Maiskomitee (2020) Beschreibung der Ernteprodukte. [Description of harvest products, In German]. https://www.maiskomitee.de/Verwertung/Tierische_ Veredlung/Beschreibung_der_Ernteprodukte. Accessed 16 June 2020

Döll S, Dänicke S (2011) The Fusarium toxins deoxynivalenol (DON) and zearalenone (ZON) in animal feeding. Prevent Vet Med Clin 102:132-145. https://doi.org/10.1016/j. prevetmed.2011.04.008

Eller MS, Robertson-Hoyt LA, Payne GA, Holland JB (2008) Grain yield and Fusarium ear rot of maize hybrids developed from lines with varying levels of resistance. Maydica 53:231-237

Emrich K, Wilde F, Miedaner T, Piepho HP (2008) REML approach for adjusting the Fusarium head blight rating to a phenological date in inoculated selection experiments of wheat. Theor Appl Genet 117:65-73. https://doi.org/10. 1007/s00122-008-0753-z

Endelman JB (2011) Ridge regression and other kernels for genomic selection with R package rrBLUP. Plant Genome J 4:250-255. https://doi.org/10.3835/plantgenome2011. 08.0024

Endelman JB, Jannink J (2012) Shrinkage estimation of the realized relationship matrix. G3 Genes Genom Genet 2:1405-1413. https://doi.org/10.1534/g3.112.004259

European Commission (2006) Commission recommendation (EC) No 576/2006 on the presence of deoxynivalenol, zearalenone, ochratoxin A, T-2 and HT-2 and fumonisins in products intended for animal feeding. https://eur-lex. 
europa.eu/LexUriServ/LexUriServ.do?uri=OJ:L:2006:

229:0007:0009:EN:PDF Accessed 7 Dec 2020

Gaikpa DS, Miedaner T (2019) Genomics-assisted breeding for ear rot resistances and reduced mycotoxin contamination in maize: methods, advances and prospects. Theor Appl Genet 132:2721-2739. https://doi.org/10.1007/s00122019-03412-2

Ganal MW, Durstewitz G, Polley A et al (2011) A large maize (Zea mays L) SNP genotyping array: development and germplasm genotyping, and genetic mapping to compare with the B73 reference genome. PLoS ONE 6(12):e28334. https://doi.org/10.1371/journal.pone.0028334

Garin V, Wimmer V, Mezmouk S et al (2017) How do the type of QTL effect and the form of the residual term influence QTL detection in multi-parent populations? A case study in the maize EU-NAM population. Theor Appl Genet 130:1753-1764. https://doi.org/10.1007/s00122-0172923-3

Garin V, Wimmer V, Borchardt D et al (2018) MppR: multiparent population qtl analysis

Gendloff EH, Rossman EC, Casale WL, Isleib TG, Hart LP (1986) Components of resistance to Fusarium ear rot in field corn. Phytopathology 76:684-688

Gilmour AR, Gogel BJ, Cullis BR, Thompson R (2009) ASReml user guide release 3.0. VSN Int Ltd, Hemel Hempstead, HP1 1ES, UK 275. https://doi.org/10.1017/ CBO9781107415324.004

Giomi GM, Kreff ED, Iglesias J et al (2016) Quantitative trait loci for Fusarium and Gibberella ear rot resistance in Argentinian maize germplasm. Euphytica 211:287-294. https://doi.org/10.1007/s10681-016-1725-z

Hallauer AR, Carena MJ, Miranda Filho JB (2010) Quantitative genetics in maize breeding. Springer, Ames

Han S, Utz HF, Liu W et al (2016) Choice of models for QTL mapping with multiple families and design of the training set for prediction of Fusarium resistance traits in maize. Theor Appl Genet 129:431-444. https://doi.org/10.1007/ s00122-015-2637-3

Han S, Miedaner T, Utz HF et al (2018) Genomic prediction and GWAS of Gibberella ear rot resistance traits in dent and flint lines of a public maize breeding program. Euphytica 214:1-20. https://doi.org/10.1007/s10681-017-2090-2

Juroszek P, von Tiedemann A (2013) Climatic changes and the potential future importance of maize diseases: a short review. J Plant Dis Prot 120:49-56. https://doi.org/10. 1007/BF03356454

Kebede AZ, Woldemariam T, Reid LM, Harris LJ (2016) Quantitative trait loci mapping for Gibberella ear rot resistance and associated agronomic traits using genotyping-by-sequencing in maize. Theor Appl Genet 129:17-29. https://doi.org/10.1007/s00122-015-2600-3

Löffler M, Miedaner T, Kessel B, Ouzunova M (2009) Mycotoxin accumulation and corresponding ear rot rating in three maturity groups of European maize inoculated by two Fusarium species. Euphytica 174:153-164. https://doi.org/ 10.1007/s10681-009-0080-8

Löffler M, Kessel B, Ouzunova M, Miedaner T (2010) Population parameters for resistance to Fusarium graminearum and Fusarium verticillioides ear rot among large sets of early, mid-late and late maturing European maize (Zea mays L.) inbred lines. Theor Appl Genet 120:1053-1062. https://doi.org/10.1007/s00122-009-1233-9

Martin M (2012) Genetic analysis of resistance to ear rot and mycotoxin contamination caused by Fusarium graminearum in European maize. $\mathrm{PhD}$ thesis, Univ. of Hohenheim, Stuttgart, Germany. https://opus.uni-hohenheim.de/ volltexte/2012/694/pdf/Diss_M_Martin.pdf Accessed 7 Dec 2020

Martin M, Miedaner T, Dhillon BS et al (2011) Colocalization of QTL for Gibberella ear rot resistance and low mycotoxin contamination in early European maize. Crop Sci 51:1935-1945. https://doi.org/10.2135/cropsci2010.11. 0664

Martin M, Dhillon BS, Miedaner T, Melchinger AE (2012a) Inheritance of resistance to Gibberella ear rot and deoxynivalenol contamination in five flint maize crosses. Plant Breed 131:28-32. https://doi.org/10.1111/j.14390523.2011.01908.x

Martin M, Miedaner T, Schwegler DD et al (2012b) Comparative quantitative trait loci mapping for Gibberella ear rot resistance and reduced deoxynivalenol contamination across connected maize populations. Crop Sci 52:32-43. https://doi.org/10.2135/cropsci2011.04.0214

Martin M, Schipprack W, Miedaner T et al (2012c) Variation and covariation for Gibberella ear rot resistance and agronomic traits in testcrosses of doubled haploid maize lines. Euphytica 185:441-451. https://doi.org/10.1007/ s10681-012-0623-2

Mesterházy A, Lemmens M, Reid LM (2012) Breeding for resistance to ear rots caused by Fusarium spp. in maize-a review. Plant Breed 131:1-19. https://doi.org/10.1111/j. 1439-0523.2011.01936.x

Miedaner T, Han S, Kessel B et al (2015) Prediction of deoxynivalenol and zearalenone concentrations in Fusarium graminearum inoculated backcross populations of maize by symptom rating and near-infrared spectroscopy. Plant Breed 134:529-534. https://doi.org/10.1111/pbr. 12297

Money D, Gardner K, Migicovsky Z, Schwaninger H, Zhong GY, Myles S (2015) LinkImpute: fast and accurate genotype imputation for nonmodel organisms. G3 Genes Genom Genet 5(11):2383-2390. https://doi.org/10.1534/ g3.115.021667

Munkvold GP (2003) Epidemiology of Fusarium diseases and their mycotoxins in maize ears. Eur J Plant Pathol. https:// doi.org/10.1023/A:1026078324268

Munkvold GP, Mcgee DC, Carlton WM (1997) Importance of different pathways for maize kernel infection by Fusarium moniliforme. Phytopathology 87:209-217. https://doi.org/ 10.1094/PHYTO.1997.87.2.209

Nelson R, Wiesner-Hanks T, Wisser R, Balint-Kurti P (2018) Navigating complexity to breed disease-resistant crops. Nat Rev Genet 19:21-33. https://doi.org/10.1038/nrg. 2017.82

Ogut F, Bian Y, Bradbury PJ, Holland JB (2015) Joint-multiple family linkage analysis predicts within-family variation better than single-family analysis of the maize nested association mapping population. Heredity 114:552-563. https://doi.org/10.1038/hdy.2014.123

Pfordt A, Ramos Romero L, Schiwek S et al (2020) Impact of environmental conditions and agronomic practices on the 
prevalence of Fusarium species associated with ear- and stalk rot in maize. Pathogens 9:236. https://doi.org/10. 3390/pathogens9030236

Piepho HP, Williams ER, Fleck M (2006) A note on the analysis of designed experiments with complex treatment structure. Hortic Sci 41:446-452

Pierron A, Alassane-Kpembi I, Oswald IP (2016) Impact of two mycotoxins deoxynivalenol and fumonisin on pig intestinal health. Porcine Health Manag 2:1-8. https://doi.org/10. 1186/s40813-016-0041-2

Poland JA, Bradbury PJ, Buckler ES, Nelson RJ (2011) Genome-wide nested association mapping of quantitative resistance to Northern leaf blight in maize. Proc Natl Acad Sci USA 108:6893-6898. https://doi.org/10.1073/pnas. 1010894108

R Development Core Team (2018) R: a language and environment for statistical computing

Ray DK, Mueller ND, West PC, Foley JA (2013) Yield trends are insufficient to double global crop production by 2050. PLoS ONE 8(6):e66428. https://doi.org/10.1371/journal. pone.0066428

Reid LM, Bolton AT, Hamilton RI et al (1992) Effect of silk age on resistance of maize to Fusarium graminearum. Can J Plant Pathol 14:293-298. https://doi.org/10.1080/ 07060669209500867

Reid LM, Hamilton RE, Mather DE (1996) Screening maize for resistance to Gibberella ear rot. Agriculture \& Agri-Food Canada, Research Branch, Eastern Cereal \& Oilseed Research Centre. Technical Bulletin (Canada, Department of Agriculture), 1996-5E

Reif JC, Hamrit S, Heckenberger M et al (2005) Trends in genetic diversity among European maize cultivars and their parental components during the past 50 years. Theor Appl Genet 111:838-845. https://doi.org/10.1007/s00122-0050004-5

Riedelsheimer C, Endelman JB, Stange M et al (2013) Genomic predictability of interconnected biparental maize populations. Genetics 194:493-503. https://doi.org/10.1534/ genetics.113.150227

Spindel JE, Begum H, Akdemir D et al (2016) Genome-wide prediction models that incorporate de novo GWAS are a powerful new tool for tropical rice improvement. Heredity 116:395-408. https://doi.org/10.1038/hdy.2015.113

Stram DO, Lee JW (1994) Variance components testing in the longitudinal mixed effects model. Biometrics 50:1171. https://doi.org/10.2307/2533455

Utz HF (2011) PlabMQTL-Software for meta-QTL analysis with composite interval mapping. Institute of Plant
Breeding, Seed Science, and Population Genetics. Univ. Hohenheim, Stuttgart, Germany

Utz HF, Melchinger AE, Schön CC (2000) Bias and sampling error of the estimated proportion of genotypic variance explained by quantitative trait loci determined from experimental data in maize using cross validation and validation with independent samples. Genetics 154:1839-1849. https://doi.org/10.2307/1403680

Vigier B, Reid LM, Dwyer LM et al (2001) Maize resistance to Gibberella ear rot: symptoms, deoxynivalenol, and yield. Can J Plant Path 23:99-105. https://doi.org/10.1080/ 07060660109506915

Whittaker JC, Thompson R, Denham MC (2000) Marker-assisted selection using ridge regression. Genet Res 75:249-252

Wisser RJ, Balint-Kurti PJ, Nelson RJ (2006) The genetic architecture of disease resistance in maize: a synthesis of published studies. Phytopathology 96:120-129. https://doi. org/10.1094/PHYTO-96-0120

Würschum T, Liu W, Gowda M et al (2012) Comparison of biometrical models for joint linkage association mapping. Heredity 108:332-340. https://doi.org/10.1038/hdy.2011. 78

Yu J, Pressoir G, Briggs WH et al (2006) A unified mixed-model method for association mapping that accounts for multiple levels of relatedness. Nat Genet 38:203-208. https://doi. org/10.1038/ng1702

Zeng ZB (1993) Theoretical basis for separation of multiple linked gene effects in mapping quantitative trait loci. Proc Natl Acad Sci USA 90(23):10972-10976. https://doi.org/ 10.1073/pnas.90.23.10972

Zeng ZB (1994) Precision mapping of quantitative trait loci. Genetics 136(4):1457-1468. https://doi.org/10.1016/j. exger.2011.07.003

Zhao Y, Mette MF, Gowda M et al (2014) Bridging the gap between marker-assisted and genomic selection of heading time and plant height in hybrid wheat. Heredity 112:638-645. https://doi.org/10.1038/hdy.2014.1

Zila CT, Samayoa LF, Santiago R et al (2013) A genome-wide association study reveals genes associated with Fusarium ear rot resistance in a maize core diversity panel. G3 Genes Genom Genet 3:2095-2104. https://doi.org/10.1534/g3. 113.007328

Publisher's Note Springer Nature remains neutral with regard to jurisdictional claims in published maps and institutional affiliations. 